\title{
The Modelling Process of a Paper Folding Problem in GeoGebra 3D ${ }^{1}$
}

\author{
Muharrem Aktumen \\ Department of Mathematics \\ Education \\ AhiEvran University \\ Kirsehir, Turkey
}

\author{
Bekir Kursat Doruk \\ Department of Mathematics \\ Education \\ AhiEvran University \\ Kirsehir, Turkey
}

\author{
Tolga Kabaca \\ Department of Mathematics \\ Education \\ Pamukkale University \\ Denizli, Turkey
}

\begin{abstract}
In this research; a problem situation, which requires the ability of thinking in three dimensions, was developed by the researchers. As the purpose of this paper is producing a modeling task suggestion, the problem was visualized and analyzed in GeoGebra3D environment. Then visual solution was also been supported by algebraic approach. So, the capability of creating the relationship between geometric and algebraic representations in GeoGebra was also presented in 3D sense.
\end{abstract}

Keywords-component; Modelling; GeoGebra 3D; Paper Folding.

\section{INTRODUCTION}

There are several studies on modeling the real life situations in GeoGebra [2, 3, 4, 5]. In this research, a problem situation has been suggested and modeling process in GeoGebra has been explained. Zbiek and Conner pointed out, modeling contributes to understand the fore known mathematical concepts thoroughly by demonstrating the applicability of mathematical thoughts to real life, to learn new mathematical concepts, to establish inter disciplinary relations and to both conceptual and operational development of the students studying in modeling processes [6]. Furthermore, the algebraic and geometric representations are needed to be connected in two ways $[1,7]$. That is, the modeling should present the advantage of understanding how algebraic facts effect the observed situations.

The problem can be described as follows according to the figure 1 and figure 2. Let's call the intersection point of the segment $[K L]$ and the line passing through the point $\mathrm{D}$ and parallel to segment $[O M]$ as $\mathrm{B}$ and the intersection point of the segment $[D K]$ and the line passing through the point $\mathrm{C}$ and parallel to the segment $[K L]$ as A. So, the rectangle $\mathrm{DABC}$ and the triangles $\stackrel{\Delta}{D O A}, A \stackrel{\Delta}{A K B}, \overrightarrow{B L C}$ and $\stackrel{\Delta}{C M D}$ can be obtained, such that the points $\mathrm{D}$ and $\mathrm{A}$ can be moved dynamically on the segments on which they are located. ${ }^{1}$

${ }^{1}$ A short summary of this article has been submitted to The International GeoGebra Institute Conference 2012 on 2123 September 2012 and supported by Ahi Evran University.

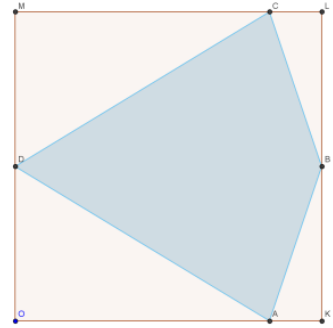

Figure 1: Statement of pre-folding

The problem was stated as "for which locations of the dynamic points $\mathrm{A}$ and $\mathrm{B}$, the segments $\left[O_{t} L_{t}\right]$ and $\left[K_{t} M_{t}\right]$ intersect?" in the research (Figure 2). The mathematical concepts related to the solution can be summarized as algebraic approach, line equations, slope, point and vector in three dimensional space and scalar triple product. As a short result, it can be stated that at least one of the points A and B must be in the midpoint of the segments on which they are located after visualization in the environment of GeoGebra 5.0. It is expected that this kind of real situated activities are attractive for the students.

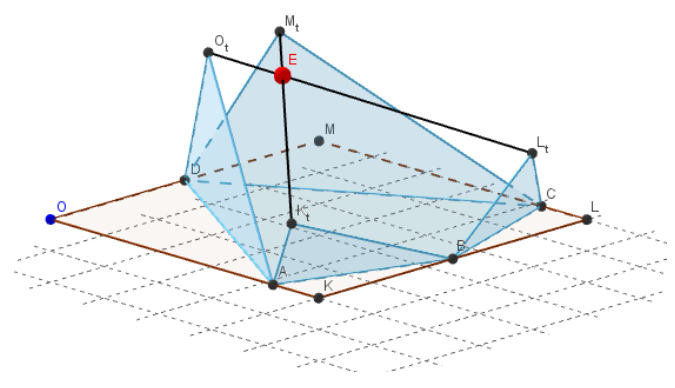

Figure 2: Statement of post-folding

\section{UNDERSTANDING THE MODEL VISUALLY}

The basic structure of the problem can be constructed as follows where the values of $\mathrm{a}, \mathrm{b}, x_{0}$ and $y_{0}$ defined as a slider tool. The points $O(0,0), K(a, 0), L(a, b), M(0, b)$ are the corners of the rectangle. The points $A\left(x_{0}, 0\right), C\left(x_{0}, b\right)$, $D\left(0, y_{0}\right)$ and $B\left(a, y_{0}\right)$, are dynamic points which are located on the sides of the rectangle and controlled by the sliders dynamically. 


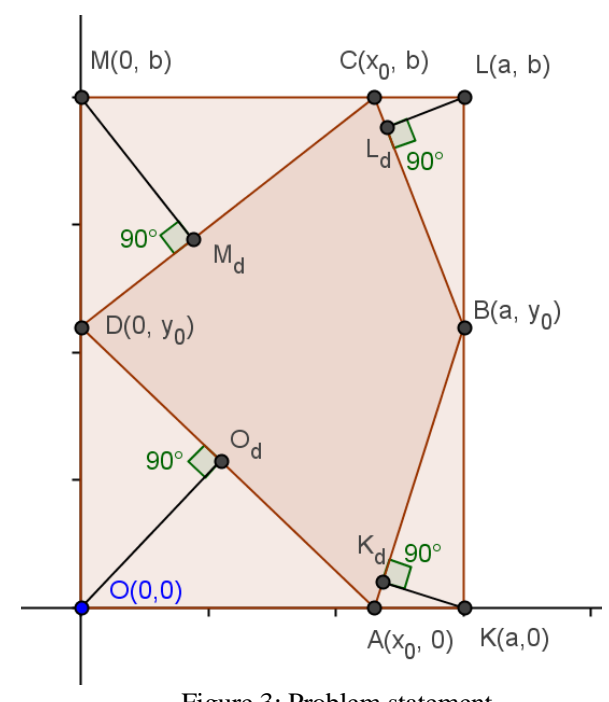

Figure 3: Problem statement

\section{ANALYZING THE Model Algebraically}

After the triangles $D O A, A K B, B L C$ and $C M D$ are folded on the sides $\mathrm{DA}, \mathrm{AB}, \mathrm{BC}$ and $\mathrm{DC}$ respectively, the coordinates of the points $O_{d}, K_{d}, L_{d}$ and $M_{d}$ must be determined.

Finding the coordinate of the point $O_{d}$ :

The intersection point of the line $d_{D A}$ and the line $d_{O O_{d}}$ will provide us to determine the coordinates of the point $O_{d}$.

Since $m_{d_{D A}}=-\frac{y_{0}}{x_{0}}$, by using the fact of the product of the tangents of two perpendicular lines, it can be obtained that $m_{d_{O O_{d}}}=\frac{x_{0}}{y_{0}}$.

Both of the lines' equations can be constructed by using a point which belongs to the line and its tangent.

The equation of the line $d_{D A}$ can be calculated as follows;

$d_{D A}: \quad y=-\frac{y_{0}}{x_{0}}\left(x-x_{0}\right) \Rightarrow y x_{0}=-y_{0} x+x_{0} y_{0}$. After the revision of equation, following form can be obtained;

$$
y x_{0}+y_{0} x=x_{0} y_{0} .
$$

The equation of the line $d_{O o_{d}}$ can be calculated as follows;

$$
d_{O o_{d}}: y=\frac{x_{0}}{y_{0}} x \Rightarrow y y_{0}=x_{0} x \text {. After the revision of }
$$
equation, following form can be obtained;

$$
y y_{0}-x_{0} x=0 .
$$

By finding the common solution of the equation (1) and (2) the coordinates of $O_{d}$ can be obtained as follows;

$$
O_{d}\left(\frac{x_{0} y_{0}^{2}}{x_{0}^{2}+y_{0}^{2}}, \frac{x_{0}^{2} y_{0}}{x_{0}^{2}+y_{0}^{2}}\right)
$$

Finding the coordinates of the point $K_{d}$ :

The intersection point of the line $d_{A B}$ and the line $d_{K K_{d}}$ will provide us to determine the coordinates of the point $K_{d}$.

Since $m_{d_{A B}}=\frac{y_{0}}{a-x_{0}}$, by using the fact of the product of the tangents of two perpendicular lines, it can be obtained that $m_{d_{K K_{d}}}=-\frac{a-x_{0}}{y_{0}}$.

Both of the lines' equations can be constructed by using a point which belongs to the line and its tangent.

The equation of the line $d_{A B}$ can be calculated as follows;

$$
\begin{aligned}
d_{A B}: y=\frac{y_{0}}{a-x_{0}}\left(x-x_{0}\right) & \Rightarrow y\left(a-x_{0}\right)=y_{0}\left(x-x_{0}\right) . \text { After } \\
& \Rightarrow y a-y x_{0}=y_{0} x-x_{0} y_{0}
\end{aligned}
$$

the revision of equation, following form can be obtained;

$$
y\left(a-x_{0}\right)-y_{0} x=-x_{0} y_{0}
$$

The equation of the line $d_{K K_{d}}$ can be calculated as follows;

$$
d_{K K_{d}}: \quad y=-\frac{a-x_{0}}{y_{0}}(x-a) \Rightarrow y y_{0}=-\left(a-x_{0}\right)(x-a) \quad \text { After }
$$

the revision of equation, following form can be obtained;

$$
y y_{0}+\left(a-x_{0}\right) x=a\left(a-x_{0}\right)
$$

By finding the common solution of the equation (4) and (5) the coordinates of $K_{d}$ can be obtained as follows;

$$
K_{d}\left(\frac{y_{0}^{2} x_{0}+a\left(a-x_{0}\right)^{2}}{y_{0}{ }^{2}+\left(a-x_{0}\right)^{2}}, \frac{y_{0}\left(a-x_{0}\right)^{2}}{y_{0}{ }^{2}+\left(a-x_{0}\right)^{2}}\right)
$$

Finding the coordinates of the point $L_{d}$ :

The intersection point of the line $d_{B C}$ and the line $d_{L L_{d}}$ will provide us to determine the coordinates of the point $L_{d}$.

Since $m_{d_{B C}}=\frac{y_{0}-b}{a-x_{0}}$, by using the fact of the product of the tangents of two perpendicular lines, it can be obtained that $m_{d_{L_{d}}}=-\frac{\left(a-x_{0}\right)}{y_{0}-b}$.

Both of the lines' equations can be constructed by using a point which belongs to the line and its tangent.

The equation of the line $d_{B C}$ can be calculated as follows; 


$$
\begin{aligned}
& d_{B C}: \quad y-y_{0}=\frac{y_{0}-b}{a-x_{0}}(x-a) \\
& \quad \Rightarrow\left(y-y_{0}\right)\left(a-x_{0}\right)=\left(y_{0}-b\right)(x-a)
\end{aligned}
$$

After the revision of equation, following form can be obtained;

$$
y\left(a-x_{0}\right)+x\left(b-y_{0}\right)=a\left(b-y_{0}\right)+y_{0}\left(a-x_{0}\right)
$$

The equation of the line $d_{L L_{d}}$ can be calculated as follows;

$$
\begin{aligned}
& d_{L L_{d}}: \quad y-b=-\frac{a-x_{0}}{y_{0}-b}(x-a) \\
& \quad \Rightarrow(y-b)\left(y_{0}-b\right)=-\left(a-x_{0}\right)(x-a)
\end{aligned}
$$

After the revision of equation, following form can be obtained;

$$
y\left(y_{0}-b\right)-\left(x_{0}-a\right) x=a\left(a-x_{0}\right)+b\left(y_{0}-b\right)(8)
$$

By finding the common solution of the equation (7) and (8) the coordinates of $L_{d}$ can be obtained as follows;

$$
L_{d}\left(\frac{a\left[\left(y_{0}-b\right)^{2}+\left(a-x_{0}\right)^{2}\right]-\left(a-x_{0}\right)\left(y_{0}-b\right)^{2}}{\left(a-x_{0}\right)^{2}+\left(y_{0}-b\right)^{2}}, \frac{y_{0}\left(a-x_{0}\right)^{2}+b\left(b-y_{0}\right)^{2}}{\left(a-x_{0}\right)^{2}+\left(b-y_{0}\right)^{2}}\right)
$$

Finding the coordinates of the point $M_{d}$ :

The intersection point of the line $d_{D C}$ and the line $d_{M M_{d}}$ will provide us to determine the coordinates of the point $M_{d}$.

Since $m_{d_{D C}}=\frac{b-y_{0}}{x_{0}}$, by using the fact of the product of the tangents of two perpendicular lines, it can be obtained that $m_{d_{M M_{d}}}=-\frac{x_{0}}{b-y_{0}}$.

Both of the lines' equations can be constructed by using a point which belongs to the line and its tangent.

The equation of the line $d_{D C}$ can be calculated as follows;

$$
d_{D C}: \quad y-y_{0}=\frac{b-y_{0}}{x_{0}} x \Rightarrow y x_{0}-y_{0} x_{0}=b x-x y_{0} .
$$

After the revision of equation, following form can be obtained;

$$
y x_{0}+x\left(y_{0}-b\right)=x_{0} y_{0}
$$

The equation of the line $d_{M M_{d}}$ can be calculated as follows;

$$
\begin{aligned}
d_{M M_{d}}: y-b=-\frac{x_{0}}{b-y_{0}} x & \Rightarrow(y-b)\left(b-y_{0}\right)=-x_{0} x \\
& \Rightarrow y b-y y_{0}-b^{2}+b y_{0}=-x_{0} x
\end{aligned}
$$

After the revision of equation, following form can be obtained;

$$
y\left(b-y_{0}\right)+x x_{0}=b\left(b-y_{0}\right)
$$

By finding the common solution of the equation (10) and (11) the coordinates of $M_{d}$ can be obtained as follows

$$
M_{d}\left(\frac{x_{0}\left(b-y_{0}\right)^{2}}{x_{0}^{2}+\left(b-y_{0}\right)^{2}}, \frac{x_{0}{ }^{2} y_{0}+b\left(b-y_{0}\right)^{2}}{x_{0}^{2}+\left(b-y_{0}\right)^{2}}\right)
$$

When the triangles $D O A, A K B, B L C$ and $C M D$ are folded perpendicular to the floor on the sides $\mathrm{DA}, \mathrm{AB}, \mathrm{BC}$ and $\mathrm{DC}$ respectively, the corner points $\mathrm{O}, \mathrm{K}, \mathrm{L}$ and $\mathrm{M}$ will be in their new places. Let's call these revised points as $O_{t}, K_{t}, L_{t}$ and $M_{t}$ respectively. These points will be in three dimensional space and the coordinates of $O_{d}, K_{d}, L_{d}$ and $M_{d}$ will be the first two components of them. The third components of each point will be the lengths of $\left|O O_{d}\right|,\left|K K_{d}\right|$, $\left|L L_{d}\right|$ and $\left|M M_{d}\right|$ (figure-2)

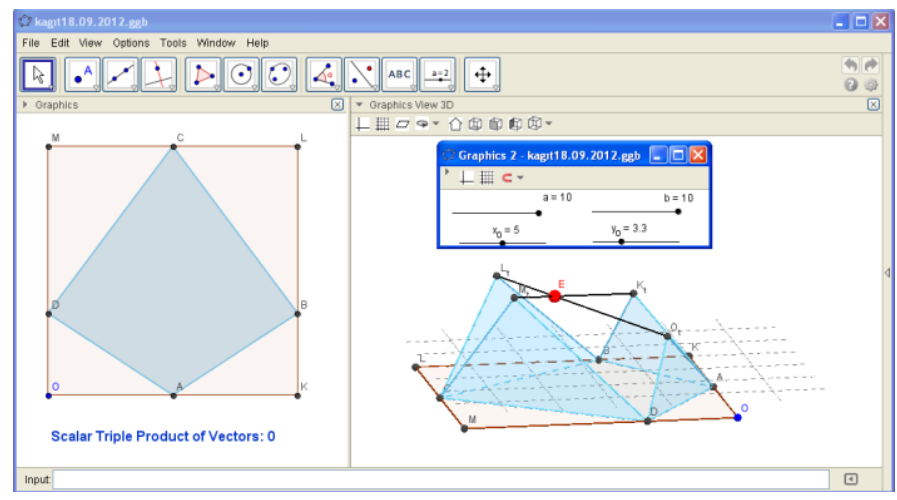

Figure 4: Comparing two and three dimensional position of folded paper.

These lengths can be calculated by using the points $\mathrm{O}, \mathrm{K}$, $\mathrm{L}$ and $\mathrm{M}$ and the equations of the lines $d_{D A}, d_{A B}, d_{B C}$ and $d_{D C}$ - After the proper calculations the lengths are found as follows;

$$
\begin{array}{ll}
\left|O O_{d}\right|=\frac{\left|x_{0} y_{0}\right|}{\sqrt{y_{0}^{2}+x_{0}^{2}}} \quad\left|K K_{d}\right|=\frac{\left|\left(a-x_{0}\right) y_{0}\right|}{\sqrt{\left(a-x_{0}{ }^{2}\right)+y_{0}^{2}}} \\
\left|L L_{d}\right|=\frac{\left|\left(a-x_{0}\right)\left(b-y_{0}\right)\right|}{\sqrt{\left(a-x_{0}{ }^{2}\right)+\left(b-y_{0}^{2}\right)}} \quad\left|M M_{d}\right|=\frac{\left|x_{0}\left(b-y_{0}\right)\right|}{\sqrt{x_{0}^{2}+\left(b-y_{0}\right)^{2}}}
\end{array}
$$

So, the ordered triples as the components of the points $O_{t}$, $K_{t}, L_{t}$ and $M_{t}$ can be stated as follows;

$$
O_{t}\left(\frac{x_{0} y_{0}^{2}}{x_{0}^{2}+y_{0}^{2}}, \frac{x_{0}^{2} y_{0}}{x_{0}^{2}+y_{0}^{2}}, \frac{\left|x_{0} y_{0}\right|}{\sqrt{y_{0}^{2}+x_{0}^{2}}}\right)
$$


$K_{t}\left(\frac{y_{0}{ }^{2} x_{0}+a\left(a-x_{0}\right)^{2}}{y_{0}{ }^{2}+\left(a-x_{0}\right)^{2}}, \frac{y_{0}\left(a-x_{0}\right)^{2}}{y_{0}^{2}+\left(a-x_{0}\right)^{2}}, \frac{\left|\left(a-x_{0}\right) y_{0}\right|}{\sqrt{\left(a-x_{0}^{2}\right)+y_{0}^{2}}}\right)$

The components of the $L_{t}=\left(L_{t_{x}}, L_{t_{y}}, L_{t_{z}}\right)$

$L_{t_{x}}=\frac{a\left[\left(y_{0}-b\right)^{2}+\left(a-x_{0}\right)^{2}\right]-\left(a-x_{0}\right)\left(y_{0}-b\right)^{2}}{\left(a-x_{0}\right)^{2}+\left(y_{0}-b\right)^{2}}$

$L_{t_{y}}=\frac{y_{0}\left(a-x_{0}\right)^{2}+b\left(b-y_{0}\right)^{2}}{\left(a-x_{0}\right)^{2}+\left(b-y_{0}\right)^{2}}$

$L_{t_{z}}=\frac{\left|\left(a-x_{0}\right)\left(b-y_{0}\right)\right|}{\sqrt{\left(a-x_{0}^{2}\right)+\left(b-y_{0}^{2}\right)}}$

$M_{t}\left(\frac{x_{0}\left(b-y_{0}\right)^{2}}{x_{0}^{2}+\left(b-y_{0}\right)^{2}}, \frac{x_{0}^{2} y_{0}+b\left(b-y_{0}\right)^{2}}{x_{0}^{2}+\left(b-y_{0}\right)^{2}}, \frac{\left|x_{0}\left(b-y_{0}\right)\right|}{\sqrt{x_{0}^{2}+\left(b-y_{0}\right)^{2}}}\right)$
Now, we have the points $O_{t}, K_{t}, L_{t}$ and $M_{t}$ in space. We can think as the mathematical question in our main problem is "what is the condition for the line segments $O_{t} L_{t}$ and $K_{t} M_{t}$ are intersected". The basic answer will be the vectors $O_{t} L_{t}$ and $K_{t} M_{t}$ must be coplanar and not parallel. Since the position of the points $O_{t}, K_{t}, L_{t}$ ve $M_{t}$ in space, we can be sure that these vectors are not parallel and has intersection points when they are reflected on the floor. Let's check the vectors $O_{t} L_{t}$ and $K_{t} M_{t}$ coplanar when they are intersected in space.

For this check, the fact of 'three vectors' scalar triple product must be zero to be coplanar" can be used. We have two vectors for this operation. By choosing the vector $O_{t} K_{t}$ additionally, we can calculate the scalar triple product.

The components of the vector $O_{t} L_{t}$;

$$
\left(\frac{a\left[\left(y_{0}-b\right)^{2}+\left(a-x_{0}\right)^{2}\right]-\left(a-x_{0}\right)\left(y_{0}-b\right)^{2}}{\left(a-x_{0}\right)^{2}+\left(y_{0}-b\right)^{2}}-\frac{x_{0} y_{0}{ }^{2}}{x_{0}{ }^{2}+y_{0}{ }^{2}}, \frac{y_{0}\left(a-x_{0}\right)^{2}+b\left(b-y_{0}\right)^{2}}{\left(a-x_{0}\right)^{2}+\left(b-y_{0}\right)^{2}}-\frac{x_{0}{ }^{2} y_{0}}{x_{0}{ }^{2}+y_{0}{ }^{2}}, \frac{\left|\left(a-x_{0}\right)\left(b-y_{0}\right)\right|}{\sqrt{\left(a-x_{0}{ }^{2}\right)+\left(b-y_{0}{ }^{2}\right)}}-\frac{\left|x_{0} y_{0}\right|}{\sqrt{y_{0}{ }^{2}+x_{0}{ }^{2}}}\right)
$$

The components of the vector $K_{t} M_{t}$;

$$
\left(\frac{x_{0}\left(b-y_{0}\right)^{2}}{x_{0}{ }^{2}+\left(b-y_{0}\right)^{2}}-\frac{y_{0}{ }^{2} x_{0}+a\left(a-x_{0}\right)^{2}}{y_{0}{ }^{2}+\left(a-x_{0}\right)^{2}}, \frac{x_{0}{ }^{2} y_{0}+b\left(b-y_{0}\right)^{2}}{x_{0}^{2}+\left(b-y_{0}\right)^{2}}-\frac{y_{0}\left(a-x_{0}\right)^{2}}{y_{0}^{2}+\left(a-x_{0}\right)^{2}}, \frac{\left|x_{0}\left(b-y_{0}\right)\right|}{\sqrt{x_{0}^{2}+\left(b-y_{0}\right)^{2}}}-\frac{\left|\left(a-x_{0}\right) y_{0}\right|}{\sqrt{\left(a-x_{0}{ }^{2}\right)+y_{0}^{2}}}\right)
$$

The components of the vector $O_{t} K_{t}$;

$$
\left(\frac{y_{0}^{2} x_{0}+a\left(a-x_{0}\right)^{2}}{y_{0}^{2}+\left(a-x_{0}\right)^{2}}-\frac{x_{0} y_{0}^{2}}{x_{0}^{2}+y_{0}^{2}}, \frac{y_{0}\left(a-x_{0}\right)^{2}}{y_{0}^{2}+\left(a-x_{0}\right)^{2}}-\frac{x_{0}^{2} y_{0}}{x_{0}^{2}+y_{0}^{2}}, \frac{\left|\left(a-x_{0}\right) y_{0}\right|}{\sqrt{\left(a-x_{0}^{2}\right)+y_{0}^{2}}}-\frac{\left|x_{0} y_{0}\right|}{\sqrt{y_{0}^{2}+x_{0}^{2}}}\right)
$$

The triple product of the vectors can be calculated by following determinant;

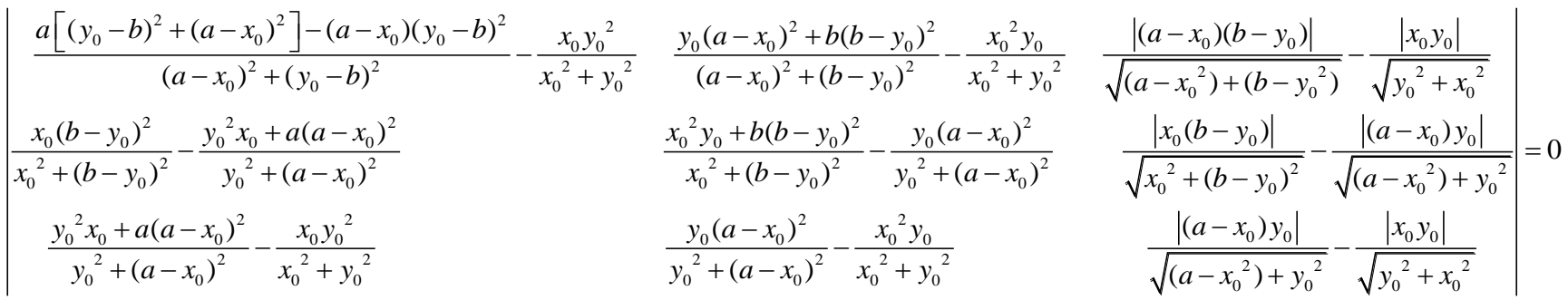

\section{CONCLUSION}

In the GeoGebra 5.0 Beta release platform, it has been easily seen that the scalar triple product is zero when the intersected position of the vectors $O_{t} L_{t}$ and $K_{t} M_{t}$ is captured. By this way, students may understand the relationship of their fore known mathematical knowledge and a real life situation [6].
There is also another opportunity of understanding the relationship between algebraic and geometric representations in this paper [7] although it still needs to be proved.We observed that at least one points of $\mathrm{A}$ and $\mathrm{B}$ must be the midpoint of the segment which the point belongs. This fact can be proved by solving the proper equations obtained from the scalar triple product. 


\section{REFERENCES}

[1] AKKOÇ, H. (2006). Fonksiyon Kavramının Çoklu Temsillerinin Çağrıştırdığı Kavram Görüntüleri. H.Ü. Eğitim Fakültesi Dergisi (H.U. Journal of Education). 30. 1-10

[2] AKTÜMEN, M. \& KABACA, T. (2012). Exploring the Mathematical Model Of The Thumbaround Motion by Geogebra. Technology, Knowledge and Learning. DOI: 10.1007/s10758-012-9194-5

[3] AKTUMEN, M., BALTACI, S. \& YILDIZ, A. (2011). Calculating the surface area of the water in a rolling cylinder and visualization as two and three dimensional by means of GeoGebra. International Journal of Computer Applications (www.ijcaonline.org/archives/volume25/number1/3170-4022)

[4] KABACA, T. \& AKTUMEN, M. (2010), Using Geogebra as an Expressive Modeling Tool: Discovering the Anatomy of the Cycloids
Parametric Equation, International Journal of GeoGebra The New Language For The Third Millennium, Zigotto Printing And Publishing House, Galati-Romania, Vol.1 No.1, 63-81 Issn: 2068-3227.

[5] AKTÜMEN, M. HORZUM \& T., CEYLAN, T.(2010). Önünde Engel BulunanBirKaleminUcununIzininParametrikDenklemininHesaplanması veGeogebraileGörselleştirme.9.

MatematikSempozyumuSergiveSenlikleri.KaradenizTeknikÜniversitesi, 20-22 Ekim 2010 Trabzon.

[6] ZBIEK, R. M. \& CONNER, A. (2006). Beyond motivation: Exploring mathematical modeling as a context for deepening students' understandings of curricular mathematics. Educational Studies in Mathematics, 63(1), 89-112.

[7] ÖZMANTAR, M., F., BINGÖlBALI, E. \& AKKOÇ, H. (2008). Matematikselkavramyanılgılarıveçözümönerileri.PegemAkademi. Ankara. 